Case Report

\title{
Airway Complications from an Esophageal Foreign Body
}

\author{
Ismael Garcia, ${ }^{1}$ Joseph Varon, ${ }^{2,3,4}$ and Salim Surani ${ }^{5}$ \\ ${ }^{1}$ Facultad de Medicina Tampico, Universidad Autónoma de Tamaulipas, Tampico, TAMPS, Mexico \\ ${ }^{2}$ Dorrington Medical Associates, Houston, TX, USA \\ ${ }^{3}$ Foundation Surgical Hospital, Houston, TX, USA \\ ${ }^{4}$ The University of Texas Health Science Center at Houston, Houston, TX 77030, USA \\ ${ }^{5}$ Texas A\&M University, Corpus Christi, TX 78413, USA \\ Correspondence should be addressed to Salim Surani; srsurani@hotmail.com
}

Received 3 August 2016; Revised 20 September 2016; Accepted 6 November 2016

Academic Editor: Fabio Midulla

Copyright (C) 2016 Ismael Garcia et al. This is an open access article distributed under the Creative Commons Attribution License, which permits unrestricted use, distribution, and reproduction in any medium, provided the original work is properly cited.

Introduction. Foreign body impaction (FBI) in the esophagus can be a serious condition, which can have a high mortality among children and adults, if appropriate diagnosis and treatment are not instituted urgently. $80-90 \%$ of all foreign bodies trapped in the esophagus usually pass spontaneously through the digestive tract, without any medical or surgical intervention. $10-20 \%$ of them will need an endoscopic intervention. Case Report. We hereby present a case of a large chicken piece foreign body impaction in the esophagus in a 25-year-old male with mental retardation. Patient developed hypoxemic respiratory failure requiring intubation. The removal required endoscopic intervention. Conclusions. Foreign bodies trapped in the upper gastrointestinal tract are a serious condition that can be fatal if they are not managed correctly. A correct diagnosis and treatment decrease the chances of complications. Endoscopic treatment remains the gold standard for extracting foreign body impaction.

\section{Introduction}

A foreign body impaction (FBI) in the esophagus can be a serious condition with high mortality rate among children and adults. A foreign body can be defined as the presence of any object, food, or material in the upper gastrointestinal tract, swallowed by accident or intentionally [1]. Children are more commonly affected by these conditions than adults. In the adult population, certain special conditions, such as mental retardation, psychiatric disorders, alcohol intake, and demented or edentulous patients, put them in a higher risk for developing an FBI [2].

Studies have shown that $80-90 \%$ of all foreign bodies trapped in the esophagus pass spontaneously whereas the remaining $10-20 \%$ of cases will require an endoscopic intervention to remove the FBI [3].

Radiological imaging of the neck and abdomen can allow the clinician to identify the radiopaque object and complications as esophageal perforation [4]. There are various ways to achieve removal of a FBI; these include nonendoscopic methods and endoscopic methods, which include flexible endoscopy versus rigid endoscopy. The rigid endoscopy is considered the gold standard for the treatment of FBI [5]. In cases when the airway of the patients seems compromised the use of a rigid endoscopy and intubation are the best treatment option $[6,7]$. The choice by the clinician relies on the patient condition, the characteristics of the object, and the location, type, form, size of material, object or food that got impacted, and the anatomical portion of the esophagus which gets affected, and the duration of FBI episode [8].

We hereby present a case of a foreign body in the esophagus caused by a food bolus impaction with a piece of chicken in a 25-year-old male with mental retardation; patient developed hypoxemic complications which were resolved. This was managed endoscopically via using flexible video endoscope by Olympus.

\section{Case Report}

25-year-old gentlemen presented to the emergency department (ED) due to acute shortness of breath and bronchospasms after ingesting the chicken piece. Patient past 


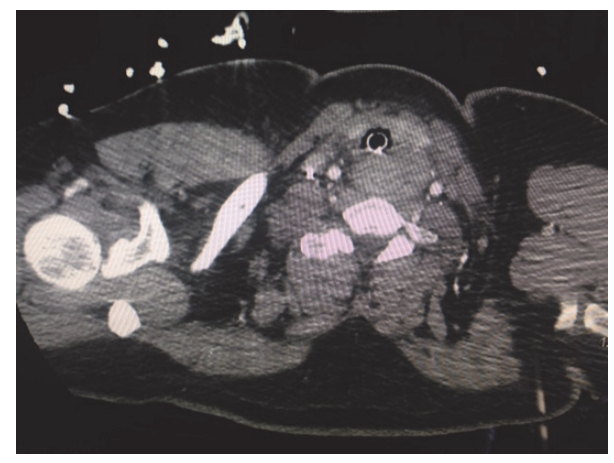

FIGURE 1: Computerized tomography of chest demonstrating foreign body impaction versus mass in esophagus.

medical history was significant for mental retardation, bipolar disorder, seizure disorder, and hypertension.

Patient was eating a chicken piece for meal, according to the witness he started to have a choking episode, Heimlich maneuver was performed, and a piece of the chicken was expelled. He started having severe respiratory distress after the incident and was transferred to the ED via ambulance. On arrival in ED, the patient was in significant respiratory distress. Patient was placed on oxygen supplementation with nonrebreather mask at $15 \mathrm{~L} / \mathrm{min}$. On auscultation stridor was heard in upper airway and rhonchi were heard in all lung fields. Patient blood pressure was $162 / 97 \mathrm{mmhg}$, heart rate was 103 beats/min, respiratory rate was $30 / \mathrm{min}$, and pulse oximetry showed an oxygen saturation of $100 \%$. Patient temperature was $99.3^{\circ} \mathrm{F}$. An X-ray with lateral view of the neck was performed, showing no radiopaque foreign body within the pharyngeal or laryngeal region. Cervical spine Xray to the level of $\mathrm{C} 5$ was within normal limits. Patient WBC count was $18.5 \mathrm{~mm}^{3}$. Electrolytes and electrocardiogram were within normal limits. Patient underwent an emergent bronchoscopy in the ED revealing no foreign body and normal airway. Patient continued to have respiratory distress and bronchospasms, which failed to improve postracemic epinephrine and steroid. Patient was intubated with excellent arterial blood gas (ABG) with no significant A-a gradient. Patient was also placed on empiric broad-spectrum antibiotic with piperacillin and tazobactam. In the following day, patient had an excellent oxygenation on arterial blood gas. Patient was extubated. Immediately after extubation patient went into severe respiratory distress and bronchospasm, requiring immediate reintubation.

CT scan of the chest was performed which showed a large soft tissue mass $8 \times 6 \mathrm{~cm}$ posterior to the trachea, extending to the left of midline posterior to the left thyroid lobe (Figure 1). The mass displaces the trachea anteriorly and slightly to the right. Differential diagnosis included esophageal mass versus large left thyroid versus possible nonopaque foreign body. Other findings reported were posterior right upper lobe and bilateral lower lobe consolidations.

In the view of the patient history of possible foreign body ingestion, patient underwent esophagogastroduodenoscopy (EGD). The flexible video endoscope was inserted and passed without difficulty up to the upper esophageal sphincter, where a very large piece of chicken was identified occluding the proximal esophagus and causing significant pressure on the posterior tracheal wall. A tunnel in the middle of the chicken piece was made and grabbed in pieces with a snare and eventually removed as much as possible, weakening the center piece so the rest of the piece could pass easily into the stomach. The scope was advanced into the duodenum, which was in the normal limits without any acute findings.

Following the EGD, the patient was able to be successfully extubated. Patient initially received intravenous antibiotics, which was switched to oral antibiotics, and was discharged home on oral antibiotics in 3 days.

\section{Discussion}

The foreign body impaction (FBI) is considering an emergent situation. FBI is defined as the presence of any object, material, or food that gets trapped in the upper gastrointestinal tract, usually swallowed by accident or in some cases, intentionally. Some data reports that around 100,000 of FBI occur each year in the United States of America [9]. This event can lead to high morbidity and mortality [10]. It is estimated that between 1,500 and 1,600 patients die yearly due to FBI and esophageal perforation being the most dreaded complication [11].

We presented a case of a FBI in a 25-year-old adult. Although children's are the ones most commonly affected (specially between 6 and 72 months of age), [6,10-12], our patient with history of mental retardation made him a highrisk person for FBI. In addition, other factors for adults include gastrointestinal alterations $[9,12,13]$, psychiatric disorders, alcohol/drug intoxication, being edentulous elderly, baseline dementia, or altered mental status $[8,11]$.

Numerous objects and food can get impacted in the upper gastrointestinal tract $[6,10,11,14]$. In our case a piece of chicken was swallowed. The literature reveals that in adults FBI with food occurs more frequently, especially meat products, fish, or chicken bones [15]. Among the pediatric population, coins and small batteries are the most common objects $[6,14]$.

Majority of the FBIs do not need any kind of intervention or treatment, data reports that around 80 and $90 \%$ of the FBIs will pass from the esophagus to the stomach without any intervention, the remaining $10-20 \%$ will need endoscopic intervention, and $1 \%$ of the FBIs cases will require surgical intervention $[6,8,10,14,15]$. In our case the need of endoscopic intervention was needed as it compromised the airway by extrinsic pressure on the membranous wall of the trachea, leading to the tracheal collapse. The clinical presentation as seen in our patient causing airway compromise is seen in $10 \%$ of the cases [16]. In some cases, patients with FBI may be asymptomatic, to be diagnosed later on during imaging studies or examination as an incidental finding. In other circumstances, patient presents with array of symptoms (see Table 1) $[11,14,16]$.

In most cases making an accurate diagnosis is simple, as patient presents with the history or has been witnessed, and other times it can be complex, especially in case of very 
TABLE 1: Clinical manifestation of foreign body impaction.

\begin{tabular}{ll}
\hline System & Symptoms \\
\hline & (i) Abdominal pain \\
& (ii) Dysphagia \\
& (iii) Halitosis \\
(iv) Hematemesis & (v) Nausea \\
Gastrointestinal & (vi) Odynophagia \\
& (vii) Regurgitation \\
& (viii) Vomiting \\
\hline & (i) Cough \\
& (ii) Drooling \\
Respiratory & (iii) Dyspnea \\
& (iv) Stridor \\
& (v) Wheezing \\
\hline
\end{tabular}

small children or adult with dementia or mental retardation that may not be able to provide adequate history. In our case, the information presented was provided by the family members of our patient and a choking episode with the chicken piece was witnessed. When ingestion of a foreign body is suspected, either by symptoms, when present, or by clinical history imaging studies with X-ray of neck, chest, and abdomen may help in diagnosis [17]. We performed an X-ray with lateral view of the neck and no radiopaque foreign body was seen in the pharyngeal or laryngeal region, as literature suggests $[18,19]$. In the event of the negative $\mathrm{X}$ ray and patient with a high index of suspicion for FBI, we performed a chest computerized tomography as our next step for diagnosis, which reported the presence of a mass posterior to the trachea, as mentioned above.

In any case of FBI the first line of treatment is to protect the airway [8]. Our patient presented with symptoms of respiratory distress and was intubated. The management used in our case was the flexible video endoscopic removal of the foreign body. The flexible endoscopy which is readily available and can be done at the bedside was the first choice. Though rigid scope can be used instead or in the cases when flexible scope fails to remove the FBI [14]. Minor complications have been reported in literature when endoscopic methods are performed [19]. The chicken piece in our patient was successfully removed with a flexible endoscope and patient then was successfully extubated and discharged home. Regardless of the methodology used after removal of the foreign body, follow-up imaging studies need to be done to ensure complete removal and rule out any complication.

FBI, though having a low complication rate, error in diagnosis or the delay in the management of these situations can lead to very critical and life treating situations. The most severe of them is perforation of the esophageal wall, which can lead to mediastinitis, abscess, fistula formation, empyema, sepsis, and death [11, 13, 15, 20-22]. Other life treating complications are airway compromise as occurred in our patient, which was treated initially by endotracheal intubation and later endoscopic removal of the FBI. Other complications from FBI are direct damage to the esophageal wall and migration of foreign body to trachea or mediastinum $[6,18]$.

\section{Conclusions}

Foreign bodies trapped in the upper gastrointestinal tract are a serious condition that can be fatal if not managed correctly. Accurate diagnosis and urgent treatment decrease the complications risk. Although majority of these events resolve spontaneously by themselves, some do require intervention. Endoscopic treatment remains as the standard for extracting foreign body impaction. Physicians need to perform adequate history and if unavailable or in doubt imaging studies need to be done to identify the FBI and site of impaction.

\section{Competing Interests}

The authors declare that there are no competing interests.

\section{References}

[1] K. H. Hong, Y. J. Kim, J. H. Kim, S. W. Chun, H. M. Kim, and J. H. Cho, "Risk factors for complications associated with upper gastrointestinal foreign bodies," World Journal of Gastroenterology, vol. 21, no. 26, pp. 8125-8131, 2015.

[2] M. H. Emara, E. M. Darwiesh, M. M. Refaey, and S. M. Galal, "Endoscopic removal of foreign bodies from the upper gastrointestinal tract: 5-year experience," Clinical and Experimental Gastroenterology, vol. 7, no. 1, pp. 249-253, 2014.

[3] P. Heger, T. F. Weber, J. Rehm, A. Pathil, F. Decker, and P. Schemmer, "Cervical esophagotomy for foreign body extraction-case report and comprehensive review of the literature," Annals of Medicine and Surgery, vol. 7, pp. 87-91, 2016.

[4] S. O. Ikenberry, T. L. Jue, M. A. Anderson et al., "Management of ingested foreign bodies and food impactions," Gastrointestinal Endoscopy, vol. 73, no. 6, pp. 1085-1091, 2011.

[5] A. Burgos, L. Rabago, and P. Triana, "Western view of the management of gastroesophageal foreign bodies," World Journal of Gastrointestinal Endoscopy, vol. 8, no. 9, pp. 378-384, 2016.

[6] F. I. Wahid, H. U. Rehman, and I. A. Khan, "Management of foreign bodies of upper digestive tract," Indian Journal of Otolaryngology and Head and Neck Surgery, vol. 66, no. 1, pp. 203206, 2014.

[7] M. Birk, P. Bauerfeind, P. H. Deprez et al., "Removal of foreing bodies on the upper gastrointestinal tract in adults: European society of gastrointestinal endoscopy (ESGE) clinical guideline," Endoscopy, vol. 48, pp. 1-8, 2016.

[8] C.-W. Hung, S.-C. Hung, C. J. Lee, W.-H. Lee, and K. H. Wu, "Risk factors for complications after a foreign body is retained in the esophagus," The Journal of Emergency Medicine, vol. 43, no. 3, pp. 423-427, 2012.

[9] R. E. Kramer, D. G. Lerner, T. Lin et al., "Management of ingested foreign bodies in children: a clinical report of the NASPGHAN endoscopy committee," Journal of Pediatric Gastroenterology and Nutrition, vol. 60, no. 4, pp. 562-574, 2015.

[10] D. Antoniou and G. Christopoulos-Geroulanos, "Management of foreign body ingestion and food bolus impaction in children: a retrospective analysis of 675 cases," Turkish Journal of Pediatrics, vol. 53, no. 4, pp. 381-387, 2011.

[11] B. Erbil, M. A. Karaca, M. A. Aslaner et al., "Emergency admissions due to swallowed foreign bodies in adults," World Journal of Gastroenterology, vol. 19, no. 38, pp. 6447-6452, 2013.

[12] S. L. W. Sperry, S. D. Crockett, C. B. Miller, N. J. Shaheen, and E. S. Dellon, "Esophageal foreign-body impactions: epidemiology, 
time trends, and the impact of the increasing prevalence of eosinophilic esophagitis," Gastrointestinal Endoscopy, vol. 74, no. 5, pp. 985-991, 2011.

[13] S. A. Jafari, M. Khalesi, S. Partovi, M. A. Kiani, H. Ahanchian, and H. R. Kianifar, "Ingested foreign bodies removed by lexible endoscopy in pediatric patients: a 10-year retrospective study," Iranian Journal of Otorhinolaryngology, vol. 26, no. 76, pp. 175179, 2014.

[14] S. Dereci, T. Koca, F. Serdaroğlu, and M. Akçam, "Foreign body ingestion in children," Türk Pediatri Arşivi, vol. 50, no. 4, pp. 234-240, 2015.

[15] S. Umihanic, F. Brick, and S. Hodzic, "Foreign body impaction in esophagus: experiences at Ear-Nose-Throat Clinic in Tuzla, 2003-2013," Journal of Ear, Nose and Throat, vol. 25, no. 4, pp. 214-218, 2015.

[16] T. O. Abbas, N. A. Shahwani, and M. Ali, "Endoscopic management of ingested foreign bodies in children: a retrospective review of cases, and review of the literature," Open Journal of Pediatrics, vol. 3, no. 4, pp. 428-435, 2013.

[17] A. Sanei-Moghaddam, A. Sanei-Moghaddam, and S. Kahrobaei, "Lateral soft tissue X-ray for patients with suspected fishbone in oropharynx, a thing in the past," Iranian Journal of Otorhinolaryngology, vol. 27, no. 83, pp. 459-462, 2015.

[18] H.-H. Chen, L.-X. Ruan, S.-H. Zhou, and S.-Q. Wang, "The utility of repeated computed tomography to track a foreign body penetrating the esophagus to the level of the thyroid gland," Oral Radiology, vol. 30, pp. 196-202, 2014.

[19] C.-C. Yao, I.-T. Wu, L.-S. Lu et al., "Endoscopic management of foreign bodies in the upper gastrointestinal tract of adults," BioMed Research International, vol. 2015, Article ID 658602, 6 pages, 2015.

[20] M. Shafique, S. Yaqub, E. S. Lie, V. Dahl, F. Olsbø, and O. Røkke, "New and safe treatment of food impacted in the esophagus: a single center experience of 100 consecutive cases," Gastroenterology Research and Practice, vol. 2013, Article ID 142703, 4 pages, 2013.

[21] C.-C. Tseng, T.-Y. Hsiao, and W.-C. Hsu, "Comparison of rigid and flexible endoscopy for removing esophageal foreign bodies in an emergency," Journal of the Formosan Medical Association, vol. 115, no. 8, pp. 639-644, 2016.

[22] D. Shreshtha, K. Sikka, C. A. Singh, and A. Thakar, "Foreign body esophagus: when endoscopic removal fails," Indian Journal of Otolaryngology and Head \& Neck Surgery, vol. 65, no. 4, pp. 380-382, 2013. 


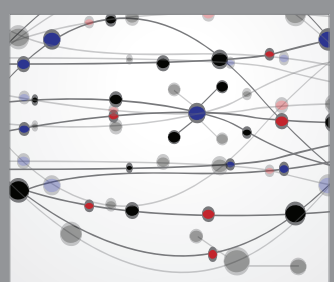

The Scientific World Journal
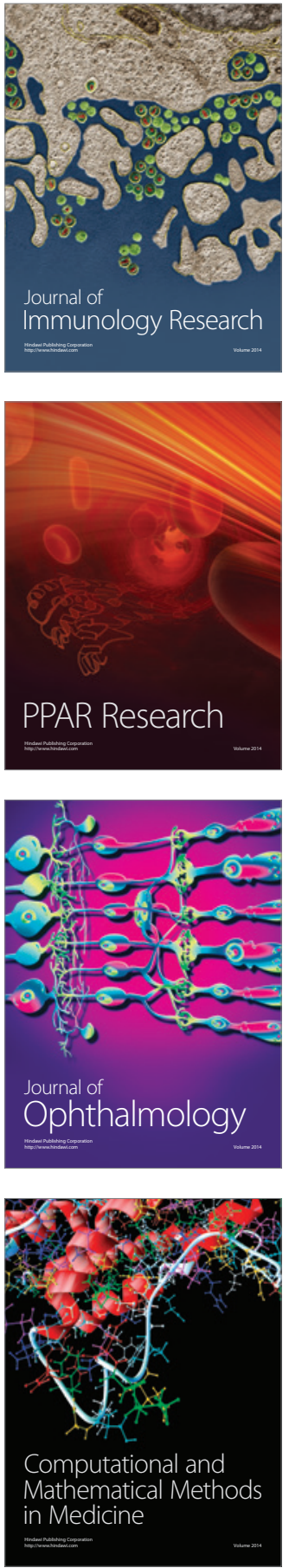

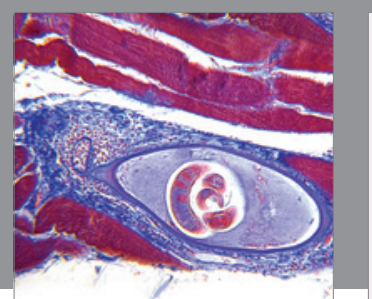

Gastroenterology Research and Practice

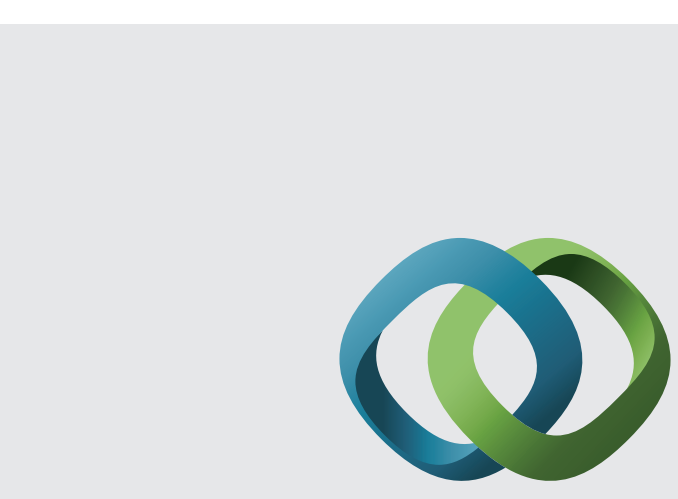

\section{Hindawi}

Submit your manuscripts at

http://www.hindawi.com
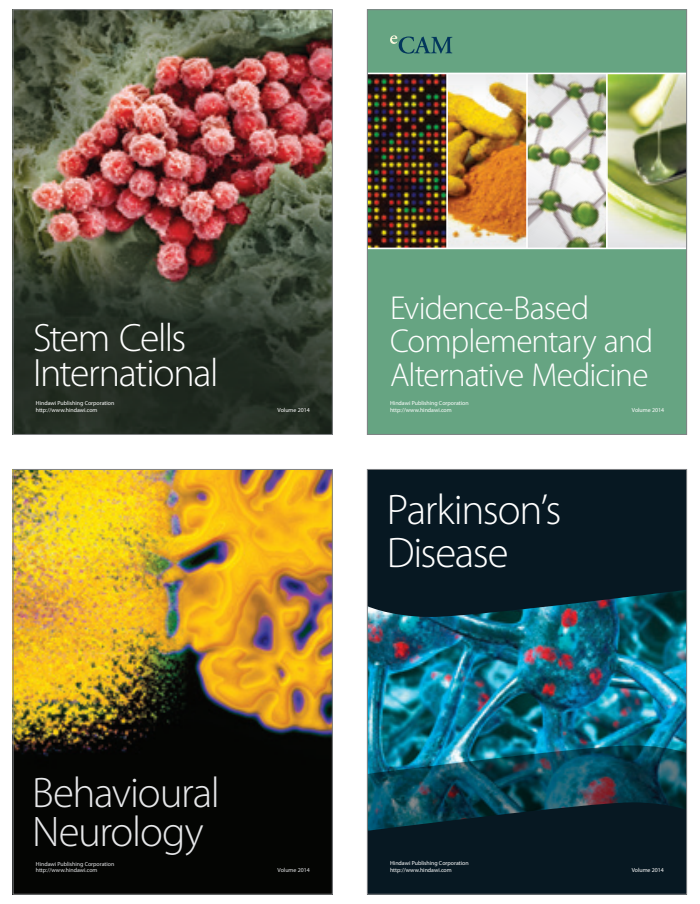
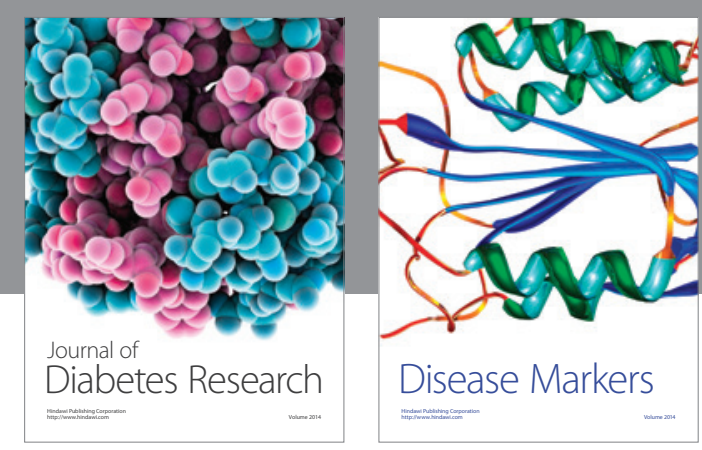

Disease Markers
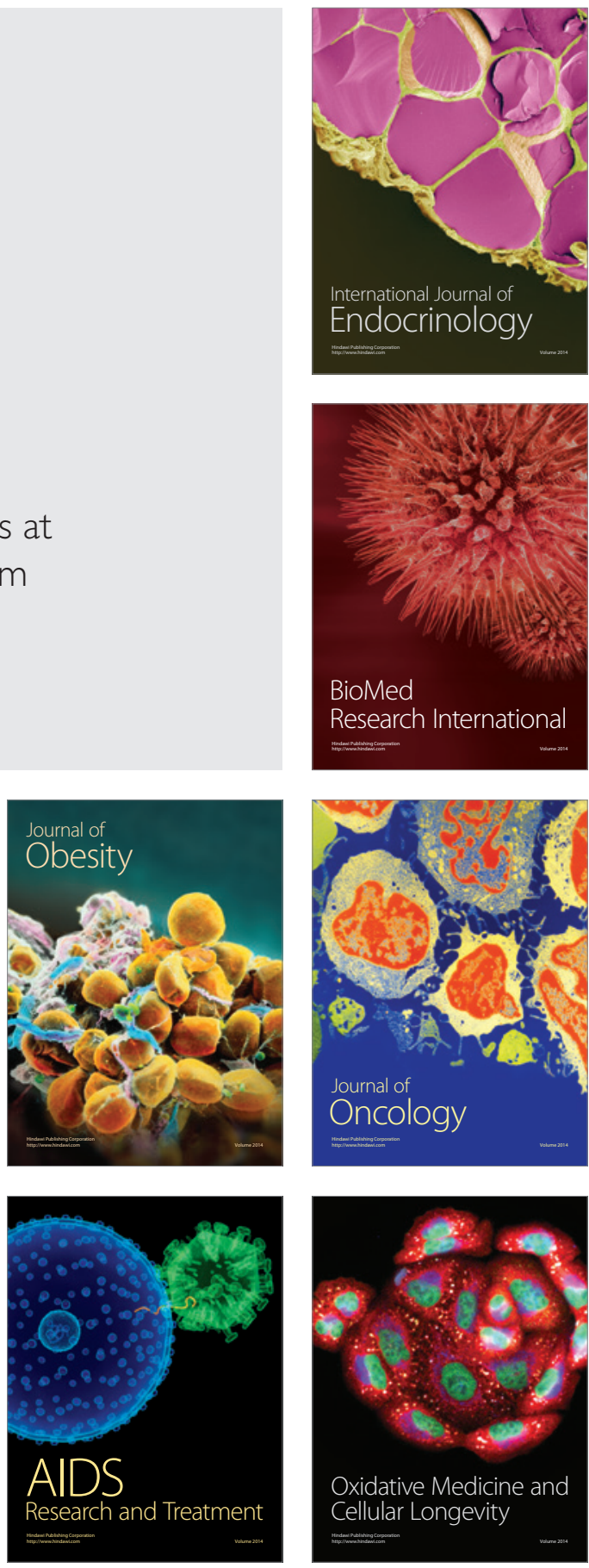